\section{Bluetongue virus antibodies in wild red deer in southern Belgium}

SIR, - Bluetongue virus serotype 8 (BTV8 ) emerged for the first time in northern Europe during summer 2006 in the Belgian/Dutch/German border region.
The virus has subsequently spread over a large area including the Netherlands, Belgium, Luxembourg, Germany, France, the UK, Denmark, Switzerland and the Czech Republic. Measures enforcing restrictions of animal movements have been put in place, vector control measures have been instigated, and vaccination will be used in an attempt to control the spread of BTV-8.

As an exhaustive knowledge of the virus biology is needed in this context, we took advantage of the existing Wallonia wildlife disease surveillance network to address the question of the possible involvement of wild ruminants in the epidemiology of the disease. Through an active surveillance programme, a total of 1182 serum samples from apparently healthy, hunter-killed red deer (Cervus elaphus) were collected during the autumns of $2005(\mathrm{n}=200)$, $2006(n=469)$ and $2007(n=513)$. The animals originated from 25 of the 37 district forests known to shelter wild cervids in southern Belgium.

The sera were examined for anti-BTV antibodies using the ID Screen Bluetongue Competition assay (ID Vet) according to the manufacturer's instructions. All sera taken in 2005 were negative but seroconversion was detected in 2006 and 2007; the apparent seroprevalence was 0.9 per cent in 2006 and 40.4 per cent in 2007. Meanwhile, 118 (2005), 130 (2006) and 133 (2007) wild cervids were found dead and recorded in a passive surveillance programme, suggesting that spontaneous mortality remained stable over the period, despite the dissemination of the virus over the entire region in 2007. The same people collected these dead animals throughout the time period so no recruitment bias should be expected.

Our results suggest that free-ranging red deer are susceptible to infection by the current BTV-8, which does not result in a significant excess mortality. The epidemiological modelling of the current BTV-8 spread, persistence and overwintering should take this aspect into account. With respect to European red deer populations, it is of crucial importance to determine whether BTV-8 infections affect fertility and/or the viability of fawns.

\section{Annick Linden,}

Bénédicte Mousset, Fabien Grégoire, David Hanrez,

Network of Wildlife Diseases, Department of Infectious and Parasitic Diseases, Faculty of Veterinary Medicine, University of Liège, Belgium

Frank Vandenbussche, Elise Vandemeulebroucke, Tine Vanbinst, Bart Verheyden, Kris de Clerck, Veterinary and Agrochemical Research Centre, Department of Virology,

Groeselenberg 99, B-1180 Brussels, Belgium 